\title{
Study Design - the First Step for a Successful Research
}

\author{
Diana Opincariu, Theodora Benedek, Manuela Chibelean
}

University of Medicine and Pharmacy, Tîrgu Mureș, Romania

\section{CORRESPONDENCE}

Theodora Benedek

Str. Gheorghe Marinescu nr. 38

540139 Tîrgu Mureș, Romania

Tel: +40 265215551

E-mail: theodora.benedek@gmail.com
Diana Opincariu • Str. Gheorghe Marinescu nr. 38 540139 Tîrgu Mureș, Romania. Tel: +40 265215551 Manuela Chibelean • Str. Gheorghe Marinescu nr. 38 540139 Tîrgu Mures, Romania. Tel: +40 265215551
One of the most difficult steps in conducting a clinical study, being of utmost importance, is the preparation of the study protocol that provides a succinct, comprehensive description of the future research. ${ }^{1}$ There are five important phases in conducting medical research studies, including planning, performance, documentation, analysis, and publication. The initial phase of any medical study consists in a precise planning, which is materialized in drafting the study protocol. ${ }^{2}$

The design of a clinical study represents a critical element for the future development of the study. A well-designed study will be easily conducted, as investigators will be guided by a well-defined set of rules in all the phases of the trial. The study protocol illustrates the procedure guidelines that are set before commencing with the research, as well as specific timelines, study population sampling, data collection and analysis, ethical considerations, methodology, and patient risks. ${ }^{3}$

The role of a research protocol is to raise the study questions and to illustrate its importance, to analyze the current knowledge on the topic, as well as to find gaps in the existing literature, which will further the study rationale and create the study hypothesis and objectives. Simultaneously, the protocol includes ethical considerations and description of the methodology used to answer the questions stated in the hypothesis, as well as discusses the prerequisites and obstacles that may or may not be encountered when achieving the objectives. The benefits derived from a well-written study design will allow the research team to plan and review the phases of the project, it will act as a guide during the study, as well as a monitor for time and budget estimates. ${ }^{1}$

A well-designed study includes non-equivocal inclusion and exclusion criteria, and a clear study protocol mentioning the procedures to be performed at baseline and during follow-up, as well as their timelines. At the same time, outcome measures and study endpoints should be presented in the protocol from the beginning, in order to make the investigators aware on all the aspects of the study.

The structure of a properly written study design includes the following six aspects: the research hypothesis, the study population, the type of study, the unit of analysis, the measuring technique, and the calculation of the sample size.

Journal of Interdisciplinary Medicine 2018;3(1):7-9

DOI: $10.2478 / \mathrm{jim}-2018-001$ 
The research hypothesis, or the questions to be answered by the study, will decide the rest of the characteristics that comprise a complete study protocol, as well as the data collection, the financial, logistical, organizational, and personnel aspects that are included in the planning of a study. The study hypothesis will also generate the objectives, which are practically answers to the questions stated after a comprehensive review of the current literature on the subject. The objective of a study should be achievable, realistic, and specific, with no ambiguous statements. Furthermore, it is advisable not to include more than two to five primary objectives, as more can lead to inaccurate results; however, researchers can include secondary objectives in their study. ${ }^{1,4,5}$ The description of the study population should include all aspects of the selection process and sample size calculation, as well as a comprehensive description of patient eligibility, exclusion and discontinuation criteria of the included subjects. ${ }^{1,3,6} \mathrm{~A}$ suitable explanation of the study type and the motives for which that study type was chosen should be given. Clinical research includes interventional (randomized clinical trials that research the clinical and pharmacological activity of certain therapeutic interventions) and non-interventional studies (these include observational studies such as case-control, cohort, cross-sectional, or ecological studies). ${ }^{7,8}$

The unit of analysis in typical clinical studies consists in the patient. However, the unit of analysis can also consist in a cell, an organ or organ system, or an animal, depending on the study population. ${ }^{3}$ The measuring technique includes both the instruments and the method that are used for the quantification of certain categorical and continuous variables that characterize the unit of analysis. Measuring instruments consist of either data collection with standardized questionnaires, such as for quality of life, or specific recordings of measuring data such as clinical or laboratory parameters. ${ }^{3}$ The measurement schedule includes the number and specific times for the measurements to be acquired, which should be standardized in order to obtain comparable results for each subject, each time, and preferably by the same investigator, thus leading to lower risk of error. ${ }^{3}$ The calculation of sample size must be conducted before the initiation of the study, as this phase ensures that the study population is large enough, but not too large. If a sample is insufficient, the power of the study will be too low, and if the sample is too large, it will bring about both ethical problems, as too many patients will be exposed to unnecessary risks, as well as financial and structural problems. ${ }^{3,9-11}$

The importance of a well-written study protocol is derived from its capacity to influence the subsequent quality of the study, the reliability of its conclusions, and the acceptance of its results for publication. The study design sets the objectives and guides the procedures, while considering possible errors and sources for research bias. Study design errors cannot be corrected after the study has been completed and once the data have been collected. ${ }^{3,12}$ Consequently, the study protocol should be precisely thought out and designed, in order to minimize the risk of errors.

A well-written study protocol should incorporate sufficient information regarding the research question, the study rationale, and the methodology, thus allowing other investigators to conduct the study and to reach comparable results. Frequent errors encountered in study protocols include insufficient detailing of the study rationale, underpowered study size sampling, invalid measurements, or unsuitable data handling and analysis. ${ }^{1}$

Publishing a study design is not always very easy. Most of the journals require prior registration of the clinical trial in an internationally recognized system, such as clinicaltrials.gov, and one of the mandatory prerequisites for publication or registration of any study design is represented by ethical approval. At the same time, publishing a study design implies the obligation of the investigators to adhere strictly to the published design, which will serve as a reference for the future publication of study results.

Recently, more journals started to publish innovative study designs. Some of them agreed to publish study designs without any peer review if the clinical study is the subject of a research grant funded by an internationally recognized body. This is conceptually possible without altering the quality of the journal, because those study designs have been already reviewed and validated by the research agencies funding the research.

Two articles published in this issue of JIM present two study designs: the INFLAMAP study and the HYBRIDHEART study. ${ }^{13,14}$ These articles present the design of two interesting studies in the field of cardiology. While INFLAMAP addresses the role of inflammation in the genesis of atrial fibrillation and its reccurence, ${ }^{13}$ HYBRIDHEART tries to develop a new tool for assessing cardiac remodeling and its complex interrelation with myocardial viability using hybrid imaging models. ${ }^{14}$ Independent of the field addressed, the design of a clinical study can represent the first step for a smooth development of the clinical study and a successful completion of the entire research.

Designing a protocol before commencing with a project is one of the most difficult stages in scientific research, as it plays a major role in the proper development of the study, as well as in obtaining reliable results. A good study protocol should be short, yet comprehensive, while providing suffi- 
cient information on its hypothesis, rationale, methodology and ethical considerations. An adequately planned research study will lead to lower rates of errors and also to results that can be published in high-quality journals.

\section{REFERENCES}

1. Al-Jundi A, Sakka S. Protocol Writing in Clinical Research. J Clin Diagn Res. 2016;10:ZE10-ZE13.

2. Altman DG, Gore SM, Gardner MJ, Pocock SJ. Statistical guidelines for contributors to medical journals. British Medical Journal (Clinical research ed). 1983;286:1489-1493

3. Röhrig B, du Prel JB, Blettner M. Study Design in Medical Research: Part 2 of a Series on the Evaluation of Scientific Publications. Deutsches Ärzteb/att International. 2009:106:184-189.

4. Röhrig B, du Prel JB, Wachtlin D, Blettner M. Types of Study in Medical Research: Part 3 of a Series on Evaluation of Scientific Publications. Deutsches Ärzteblatt International. 2009;106:262-268.

5. Thiese MS. Observational and interventional study design types; an overview. Biochemia Medica. 2014;24:199-210.
6. Zodpey SP. Sample size and power analysis in medical research. Indian J Dermato/ Venereol Leprol. 2004;70:123-128.

7. Checkoway H, Pearce N, Kriebel D. Selecting appropriate study designs to address specific research questions in occupational epidemiology. Occupational and Environmental Medicine. 2007;64:633-638.

8. Bagatin E, Miot HA. How to design and write a clinical research protocol in Cosmetic Dermatology. Anais brasileiros de dermatologia. 2013;88:6975.

9. Eng J. Sample size estimation: how many individuals should be studied? Radiology. 2003;227:309-313.

10. Halpern SD, Karlawish JH, Berlin JA. The continuing unethical conduct of underpowered clinical trials. JAMA. 2002;288:358-362.

11. Palmer CR. Ethics and statistical methodology in clinical trials. J Med Ethics. 1993;19:219-222.

12. Sackett DL. Bias in analytic research. J Chronic Dis. 1979;32:51-63.

13. Korodi S, Benedek T, Bordi L, et al. Inflammatory and Imaging-based Predictors of Atrial Fibrillation Recurrence after Pulmonary Vein Isolation Using Electroanatomical Mapping - the INFLAMAP Study. Journal of Interdisciplinary Medicine. 2018;3:10-15.

14. Stănescu A, Benedek I, Morariu M, et al. New Imaging-derived Biomarkers Based on Tridimensional CTA/MRI Hybrid Models for Complex Assessment of Myocardial Viability after Myocardial Infarction - the HYBRIDHEART Study. Journal of Interdisciplinary Medicine. 2018;3:16-20. 